\title{
A NEW PROOF OF THE ORLICZ-LORENTZ BUSEMANN-PETTY CENTROID INEQUALITY
}

\section{ZENGLE ZHANG AND NIUFA FANG}

Abstract. The Orlicz-Lorentz Busemann-Petty centroid inequality was recently established by Nguyen [16]. In this paper, using shadow systems, a new proof of the Orlicz-Lorentz BusemannPetty centroid inequality is given.

Mathematics subject classification (2010): 52A40.

Keywords and phrases: Orlicz-Lorentz centroid body, Orlicz-Lorentz Busemann-Petty centroid inequality, shadow system.

\section{REFERENCES}

[1] K. Böröczky, E. Lutwak, D. YAng, G. Zhang, The log-Brunn-Minkowski inequality, Adv. Math., 231 (2012) 1974-1997.

[2] S. CAmpi, P. Gronchi, The $L_{p}$-Busemann-Petty centroid inequality, Adv. Math., 167 (2002) 128141.

[3] F. Chen, J. Zhou, C. Yang, On the reverse Orlicz, Busemann-Petty centroid inequality, Adv. in Appl. Math., 47 (2011) 820-828.

[4] R. Gardner, The Brunn-Minkowski inequality, Bull. Amer. Math. Soc.(New Series), 39 (3) (2002) $355-405$.

[5] R. Gardner, D. Hug, W. Weil, D. Ye, The dual Orlicz-Brunn-Minkowski theory, J. Math. Anal. Appl., 430 (2015) 810-829.

[6] C. Haberl, F. Schuster, General $L_{p}$ affine isoperimetric inequalities, J. Diff. Geom., 83 (2009) $1-26$.

[7] C. Haberl, F. Schuster, Asymmetric affine $L_{p}$ Sobolev inequalities, J. Funct. Anal., 257 (2009) 641-658.

[8] J. Haddad, C. H. Jimenez, M. Montenegro, Sharp affine Sobolev type inequalities via the $L_{p}$ Busemann-Petty centroid inequality, J. Funct. Anal., 271 (2016) 454-473.

[9] Q. HuAng, B. He, On the Orlicz Minkowski problem for polytopes, Discrete Comput. Geom., 48 (2012) 281-297.

[10] A. Li, G. Leng, A new proof of the Orlicz Busemann-Petty centroid inequality, Proc. Amer. Math. Soc., 139 (2011) 1473-1481.

[11] M. Ludwig, Minkowski valuations, Trans. Amer. Math. Soc., 357 (2005) 4191-4213.

[12] E. LutwaK, The Brunn Minkowski-Firey theory II: Affine and geominimal surface areas, Adv. Math., 118 (1996) 244-294.

[13] E. LutwaK, D. YAng, G. ZHANG, $L_{p}$ affine isoperimetric inequalities, J. Diff. Geom., 56 (2000) 111-132.

[14] E. LutwaK, D. Yang, G. Zhang, Orlicz projection bodies, Adv. Math., 223 (2010) 220-242.

[15] E. Lutwak, D. YAng, G. Zhang, Orlicz centroid bodies, J. Diff. Geom., 84 (2010) 365-387.

[16] V. H. NguYEn, Orlicz-Lorentz centroid bodies, Adv. in Appl. Math., 92 (2018) 99-121.

[17] C. M. PetTy, Centroid surfaces, Pac. J. Math., 11 (1961) 1535-1547.

[18] C. M. PetTy, Ellipsoids, in “Convexity and its Applications” (P. M. Gruber and J. M. Wills, Eds.), pp. 264-276, Birkhäser, Basel, 1983.

[19] R. SchneIder, Convex Bodies: The Brunn-Minkowski Theory, Encyclopedia of Mathematics and its Applications, 44, Cambridge University Press, Cambridge, 2014. 
[20] G. C. SHEPHARD, Shadow systems of convex sets, Israel J. Math., 2 (1964) 229-236.

[21] T. WANG, The affine Sobolev-Zhang inequality on BV( $\left.\mathbb{R}^{n}\right)$, Adv. Math., 230 (2012) 2457-2473.

[22] D. XI, G. Leng, Dar's conjecture and the log-Brunn-Minkowski inequality, J. Diff. Geom., 103 (2016) 145-189.

[23] D. XI, H. Jin, G. Leng, The Orlicz Brunn-Minkowski inequality, Adv. Math., 260 (2014) 350-374.

[24] D. Ye, Dual Orlicz, Brunn Minkowski theory: Dual Orlicz, $L_{\phi}$ affine and geominimal surface areas, J. Math. Anal. Appl., 443 (2016) 352-371.

[25] G. Zhang, The affine Sobolev inequality, J. Diff. Geom., 53 (1999) 183-202.

[26] B. ZhU, S. Mou, N. FAng, The role of the form body in bounds for inclusion measures, Math. Nachr., 290 (2017) 2413-2423.

[27] B. ZHU, W. XU, Reverse Bonnesen-style inequalities on surfaces of constant curvature, Int. J. Math., 29 (2018) 1850040.

[28] B. Zhu, J. Zhou, W. Xu, Dual Orlicz-Brunn-Minkowski theory, Adv. Math., 264 (2014) 700-725.

[29] G. ZHU, The Orlicz centroid inequality for star bodies, Adv. Appl. Math., 48 (2012) 432-445.

[30] D. Zou, G. XIONG, Orlicz-John ellipsoids, Adv. Math., 265 (2014) 132-168. 